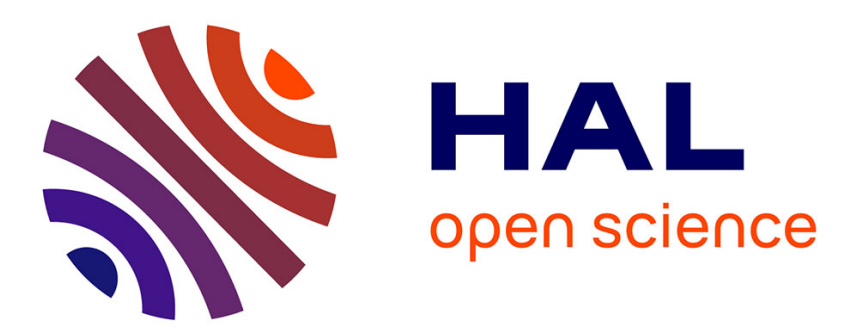

\title{
Impact of the electrostatic interaction on the diffusion of small polyelectrolytes in charged colloidal suspensions
}

Caterina Dolce, Guillaume Mériguet

\section{To cite this version:}

Caterina Dolce, Guillaume Mériguet. Impact of the electrostatic interaction on the diffusion of small polyelectrolytes in charged colloidal suspensions. Journal of Molecular Liquids, 2018, 272, pp.821-827. 10.1016/j.molliq.2018.10.07 . hal-01992063

\section{HAL Id: hal-01992063 \\ https://hal.sorbonne-universite.fr/hal-01992063}

Submitted on 24 Jan 2019

HAL is a multi-disciplinary open access archive for the deposit and dissemination of scientific research documents, whether they are published or not. The documents may come from teaching and research institutions in France or abroad, or from public or private research centers.
L'archive ouverte pluridisciplinaire HAL, est destinée au dépôt et à la diffusion de documents scientifiques de niveau recherche, publiés ou non, émanant des établissements d'enseignement et de recherche français ou étrangers, des laboratoires publics ou privés. 


\title{
Impact of the electrostatic interaction on the diffusion of small polyelectrolytes in charged colloidal suspensions
}

\author{
Caterina Dolce ${ }^{\mathrm{a}}$, Guillaume Mériguet ${ }^{*, a}$ \\ ${ }^{a}$ Sorbonne University, CNRS, Laboratoire PHENIX, Paris, France
}

\begin{abstract}
This work investigates the impact of the presence of charged obstacles, consisting of charged silica nanoparticles, on the diffusion of short polyelectrolytes, from the simple propanoate to longer polyacrylate. In the range of volume fraction studied $(\phi<0.2)$, the increase of the volume fraction of the obstacles leads to a linear decrease of the diffusion coefficient. The data show that the decrease is significantly larger than what is expected for a diffusion only restricted by the pure obstruction of the obstacles. The electrostatic origin of this reduced diffusion is confirmed by the study of the system with the addition of salt and the change of $\mathrm{pH}$, since the addition of salt screens the interaction between obstacles and diffusers, and the change of $\mathrm{pH}$ modifies their charge. To further investigate the phenomenon, the diffusion coefficient is computed using a cell-model to solve Smoluchowski equation of diffusion in the presence of a screened Coulomb potential between the diffuser and the obstacles. A semi-quantitative agreement is found between the cell-model and the experimental data. Finally, the perspectives and limitations of the model are discussed.
\end{abstract}

Key words: Polyelectrolyte, diffusion, crowding, nanoparticle, PFG-NMR, Cell-model

\section{Introduction}

The diffusion in crowded media is a challenging issue since the interactions between the diffuser, obstacles and solvent have to be considered. This phenomenon occurs in various contexts, ranging from intracellular biological processes [1-8] or porous media [9], polymer composites [10, 11], colloidal slurries or glasses [12], fuel cell membranes [13] and gels [14]. To address this issue in such complex media, the resort to a simpler model system is a valuable first step. The investigation of the dynamics of a polymer chain in a dispersion of particles acting as crowders can then be used as a first model to reveal the mechanisms at play.

To understand the dynamical behaviour of polymer-particle composites, a significant number of studies have investigated the diffusion of either the particles [15, 16] or the polymer [11, 17, 18]. In the latter studies, the polymer chains are neutral and large compared to the obstacles consisting of nanoparticles. In many situations of interest, the diffusion of rather small charged species in a dispersion of obstacles has to be considered, e.g. the diffusion of small proteins in the cytoplasm or the diffusion of small organic matter in slurries of mineral particles. The effect of the electrostatic interaction on the diffusion of such objects still poorly known. However, the interaction between the diffuser and the obstacles can significantly and non monotonously alter the dynamics of the diffuser [19, 20]. Besides, the notable effect of electrostatic interaction on the diffusion of small ions [21] and small molecules [22] has already been evidenced, but the question remains open for larger polyelectrolytes.

The aim of the present study is therefore to shed some light on the effect of the electrostatic interaction on the diffusion of small polyelectrolytes dispersed in charged colloidal dispersions. After recalling the main results concerning the diffusion of molecules or colloids in concentrated suspensions, we address the diffusion of variable size polyacrylate in suspensions of charged silica nanoparticles. We focus on the self-diffusion of polyelectrolytes small compared to the nanoparticles so that we can assume that the conformation of the chain does not significantly change while they diffuse. In this preliminary investigation, we will restrict the study to systems where the crowding is not so strong (maximum volume fraction of the obstacles the order of $\phi=0.2$ ) and explore the long-time regime by diffusion NMR [23, 24] that probes micrometer length scale, far larger than the nanoparticles size. In order to assess the effect of the electrostatic interaction, the influence of the ionic strength and of the $\mathrm{pH}$ of the solution are considered. Finally, we compare our experimental findings with a cell model developed to include the effect of the electrostatic interaction and discuss its limitations and possible valuable outcomes.

\section{Background}

The description of self-diffusion in crowded systems poses considerable theoretical problems, since this phenomenon depends on multiple parameters such as the geometry of the system, the concentration and size of the obstacles, and the interaction between the diffusers and the obstacles, either direct or indirect via hydrodynamic coupling [25-30]. In these systems, the observed decrease of the self-diffusion coefficient may be ascribed to two main mechanisms. The first mechanism is the consequence of the volume occupied by the obstacles and hence inaccessible to the diffusers. As a result of this obstruction effect, the diffusion path length is increased which leads to 
interactions between diffusers and obstacles, which may either increase or decrease the self-diffusion coefficient [19, 20].

The diffusion in concentrated colloidal dispersions has been investigated extensively by theoretical methods [32, 33] and numerical simulations such as Brownian Dynamics [34-36], Stokesian dynamics [37] and Multi-Particle Collision Dynamics [38, 39]. In the case of hard spheres without hydrodynamic interactions, a decrease of the diffusion coefficient compared to the diffusion coefficient in the absence of obstacles, $D^{\circ}$, is observed. It can be described to the first order in the volume fraction of obstacles $\phi$ as

$$
D=D^{\circ}[1-\alpha \phi]
$$

with a value of $\alpha=2$ [32, 33]. When hydrodynamic interactions are included, there is a relative enhancement of the diffusion coefficient of the order of 5 to $8 \%$ compared to computations without hydrodynamic interactions [40,-42].

While the dispersions of colloidal particles of the same type has been extensively investigated, the long-time self-diffusion of charged colloidal particles in mixtures have been hardly studied. Nägele et al. used the Generalized Langevin Equation [43-45] without hydrodynamic interaction or a modecoupling scheme that includes the effect of hydrodynamics [46]. The authors used a repulsive screened Coulomb potential, and found that if the particles bear like charges, the increase of the radius of the larger particle or the increase of the charge leads to a decrease of the diffusion of both particles. In the present systems, the diffusers are smaller than the obstacles, the small particle diffusion is then expected to exhibit a significant dependence on the large particle concentration.

The aforementioned theoretical or numerical methods can contain high complexities in terms of interaction potential but also of hydrodynamics, however their implementation and use are time consuming. In the present study, we will take advantage of the difference of diffusion coefficients between the smaller polyacrylate and the larger silica particles, and consider the latter obstacles as immobile in a first approximation.

The diffusion of small molecules or ions in a dispersions of fixed obstacles was addressed by Wang who derived an expression for the effective diffusion coefficient of solvent molecules as a function of the volume fraction of identical spheroidal obstacles to study the self-diffusion of water in protein solutions [47]. Later, with the use of cell models to solve Fick's equation [27, 48], the initial expression of Wang was corrected. Venema et al. extended the previous studies to colloidal crystals and examined the effect of crystal structure of the obstacles on the diffusion of the molecules [49]. Using another method to solve the diffusion equation, they determined the low- $\phi$ dependence of the diffusion coefficient as

$$
D=D^{\circ}\left[1-\frac{\phi}{2}+\frac{\phi^{2}}{4}-\frac{\phi^{3}}{8}+\frac{\phi^{4}}{16}\right]
$$

Below $\phi=0.2$, the high-order terms in equation 2 are smaller than $1 \%$, which is the order of magnitude of the experimental error of the method used in the present study (PFG-NMR). As a result, the behaviour of the diffusion of a small molecule in a dispersion of monodisperse uncharged obstacles is well described in this regime by a linear decrease with the volume fraction (equation 1 with $\alpha=\frac{1}{2}$.
Beyond the investigation of pure obstruction, the advantage of cell models is that an interaction potential between the diffuser and the obstacles can straightforwardly be included by solving the Smoluchowski equation. For example, Bell and Dunning [50] and later Chan and Halle [51] have used the Poisson-Boltzmann interaction potential to investigate the diffusion of counter-ions. The same method was used to investigate the counter-ion diffusion in different systems and geometries, linear polyelectrolytes [52, 53] or hydrogels [54]. To overcome the limitations of Poisson-Boltzmann for multivalent counterions, Bratko and Lindman successfully used the modified Poisson-Boltzmann (MPB) potential to describe the diffusion in highly charged micellar systems [55].

Beyond the determination of the diffusion of small polyacrylate in dispersions of charged silica particles and the assessment of the influence of the electrostatics, one of the purposes of this work is to test the applicability of a cell model description based on Bell's steady state approach [48] with a screen Coulomb potential.

\section{Experimental methods and cell model}

\subsection{Experimental}

\subsubsection{Materials}

The monomer $(n=1)$, propanoic acid (Alfa Aesar, 99\%), the dimer $(n=2)$, glutaric acid (Alfa Aesar, 99\%) and the trimer $(n=3), 1,3,5$-pentanetricarboxylic acid (TCI, > 98\%) were used as received. The longer polyelectrolytes, sodium polyacrylate or PAANa, $\left[-\mathrm{CH}_{2}-\mathrm{CH}(\mathrm{COONa})-\right]_{n}$, of varying molecular weights $\left(M_{w}=2.1\right.$ and $15 \mathrm{~kg} \mathrm{~mol}^{-1}$, respectively $n=22$ and 160 [56]) were purchased from Sigma-Aldrich. During the preparation of the samples, the concentration was corrected from the initial water content of the different products.

The nanoparticles used in this work as obstacles are silica nanoparticles. Ludox LS colloidal silica (30 wt $\%, R_{\mathrm{o}}=$ $8 \mathrm{~nm}$ ) aqueous suspensions were provided by Sigma-Aldrich. The dispersions have then been purified by a dialysis process. The commercial suspensions are loaded in Spectra/Por molecular porous membrane tubings (molecular weight cut-off: $6-8 \mathrm{~kg} \mathrm{~mol}^{-1}$, pore size $\simeq 2 \mathrm{~nm}$ ) and immersed in aqueous solutions with a weight fraction of $2 \%$ of Poly(ethylene glycol) $\left(35 \mathrm{~kg} \mathrm{~mol}^{-1}\right)$. The evolution of the purification process of silica suspensions has been monitored by measurements of the electrical conductivity of the external bath during 5 days. At the end of the process, the concentration of ions is close to $8 \times 10^{-3} \mathrm{~mol} \mathrm{~L}^{-1}$.

The stability of the polyelectrolyte-silica mixtures was checked with Dynamic Light Scattering, Laser Doppler Electrophoresis and NMR measurements which are described in the supplementary material. The short term stability of the suspensions was sufficient to perform the diffusion measurements in non-aggregated suspensions.

Titrations were performed to determine the charge of the particles and polyelectrolytes. Acid-base titrations of the polyelectrolytes and oligomers were carried out in a previous study [56]. The details of the titrations and the determination of the effective charge of the silica nanoparticles are given in the supplementary material. 


\subsubsection{Nuclear Magnetic resonance diffusion experiments (PFG-NMR)}

NMR measurements were performed on a Bruker Avance DRX 500 NMR spectrometer operating at $499.76 \mathrm{MHz}$ for ${ }^{1} \mathrm{H}$. The spectra were recorded in a $90 \% \mathrm{H}_{2} \mathrm{O}-10 \% \mathrm{D}_{2} \mathrm{O}$ mixture (residual HOD, peak at $4.7 \mathrm{ppm}$ ) for locking purpose. The sample temperature was controlled by a Bruker BCU and set to 298.0 K. A low power pre-saturation pulse of water signal was used during the recycle delay to attenuate the solvent signal. To obtain the diffusion coefficient, the pulsed field gradient (PFG) stimulated echo sequence BPP-LED (ledbpgppr2s Bruker sequence) with a water pre-saturation was used [23, 57].

The gradient pulses were sine-shaped and their duration $\delta$ was $5 \mathrm{~ms}$. The diffusion delay $\Delta$ was $0.200 \mathrm{~s}$ and the field gradient amplitude $g$ was varied with 16 increments, from $2 \%$ to $80 \%$ of the maximum amplitude $\left(57 \times 10^{-2} \mathrm{~T} \mathrm{~m}^{-1}\right)$. To obtain each spectrum, sixteen scans were accumulated and for each sample four spectra were acquired.

The measured intensity $I(b)$ depends on the diffusion coefficient $D$ and on the diffusion sensitivity factor $b, b=$ $\gamma^{2} g^{2} \delta^{2}(\Delta-\delta / 3-\tau / 2)$, where $\gamma$ is the gyromagnetic ratio and $\tau(400 \mu \mathrm{s})$ is a recovery delay after the gradient pulse. The absolute value of the gradient $g$ was calibrated with the measurement of the self-diffusion coefficient of HOD in $\mathrm{D}_{2} \mathrm{O}$ $\left(D_{\mathrm{HOD}}=1.902 \times 10^{-9} \mathrm{~m}^{2} \mathrm{~s}^{-1}[58,59]\right)$.

\subsection{Cell Model description}

In order to study the self-diffusion of ions in the electric fields of spherical particles, Bell [48] proposed to apply a weak macroscopic gradient of tagged diffusers and to solve Smoluchowski equation in the stationary state:

$$
0=\nabla^{2} c(\vec{r}, t)+\nabla[c(\vec{r}, t) \nabla \tilde{\psi}(\vec{r})]
$$

where $c$ is the concentration of the tagged diffuser and $\tilde{\psi}=$ $\psi / k_{B} T$ is the reduced interaction potential between the obstacles and the diffusers. In the present study, the diffuser can be far larger than a simple ion and the best suited interaction potential is then the screened Coulomb potential (SCP) [60]:

$$
\tilde{\psi}(r)=\ell_{B} \frac{z_{\mathrm{p}} \mathrm{e}^{\kappa R_{\mathrm{p}}}}{1+\kappa R_{\mathrm{p}}} \frac{z_{\mathrm{o}} \mathrm{e}^{\kappa R_{\mathrm{o}}}}{1+\kappa R_{\mathrm{o}}} \frac{\mathrm{e}^{-\kappa r}}{r}
$$

where $z_{i} e$ and $R_{i}$ are respectively the effective charge (see supplementary material) and the radius of the object (the indices $\mathrm{p}$ and $\mathrm{o}$ stand respectively for the polyelectrolyte diffuser and the nanoparticle obstacle), $\ell_{\mathrm{B}}=e^{2} / 4 \pi \varepsilon_{0} \varepsilon_{\mathrm{r}} k_{\mathrm{B}} T$ and $\kappa^{-1}=1 / \sqrt{8 \pi \ell_{\mathrm{B}} I}$ the Bjerrum and Debye lengths of the medium of a ionic strength $I$.

To solve Smoluchowski equation (equation 3), a spherical cell with a radius $R_{\mathrm{c}}$ is considered at a center of which a spherical charged obstacle of radius $R_{\mathrm{O}}$ is placed. The corresponding volume fraction of obstacles can then be easily evaluated as:

$$
\phi=\left(\frac{R_{\mathrm{o}}}{R_{\mathrm{c}}}\right)^{3}
$$

Bell [48] has shown that for the following boundary conditions of the interaction potential:

$$
\tilde{\psi}\left(r=R_{\mathrm{c}}\right)=0 \quad ; \quad \frac{\mathrm{d} \tilde{\psi}}{\mathrm{d} r}\left(r=R_{\mathrm{c}}\right)=0
$$

the diffusion coefficient writes

$$
\frac{D}{D^{\circ}}=\frac{\chi\left(R_{\mathrm{c}}\right)}{\left\langle\mathrm{e}^{-\tilde{\psi}}\right\rangle}=\chi\left(R_{\mathrm{c}}\right) \frac{\frac{4}{3} \pi R_{\mathrm{c}}^{3}}{\int_{R_{\mathrm{o}}}^{R_{\mathrm{c}}} \mathrm{e}^{-\tilde{\psi}(r)} 4 \pi r^{2} \mathrm{~d} r}
$$

where $\chi$ is the solution of the following first order differential equation:

$$
\begin{aligned}
r \frac{\mathrm{d} \chi}{\mathrm{d} r}+\chi(r)\left(1+\chi(r)-r \frac{\mathrm{d} \tilde{\psi}}{\mathrm{d} r}\right) & =2 \\
\chi\left(R_{\mathrm{o}}\right) & =0
\end{aligned}
$$

The boundary condition (equation 9) comes from the impenetrability of the obstacle which corresponds to a zero flux condition at this boundary. Unfortunately, there are only a limited number of cases for which either the function $\chi$ or the integral $\langle\exp (-\tilde{\psi})\rangle$ exhibit an analytical expression apart from the hard sphere case. Equation 8 was then solved numerically by the Fehlberg fourth-fifth order Runge-Kutta method, with degree four interpolant using Maple ${ }^{\mathrm{TM}}$ software [61].

\section{Results and Discussion}

In highly concentrated or porous media, the self-diffusion may become complex and exhibit unusual behaviors [62-64]. The dependence on time of the mean squared displacement can either deviate from linearity (anomalous diffusion) or display more than one linear regimes in the case of diffusers being temporally trapped in pores. For the systems under study, only usual Gaussian diffusion was observed. This was checked by varying the diffusion delay $\Delta$ in the NMR pulse sequence.

\subsection{Effect of the volume fraction}

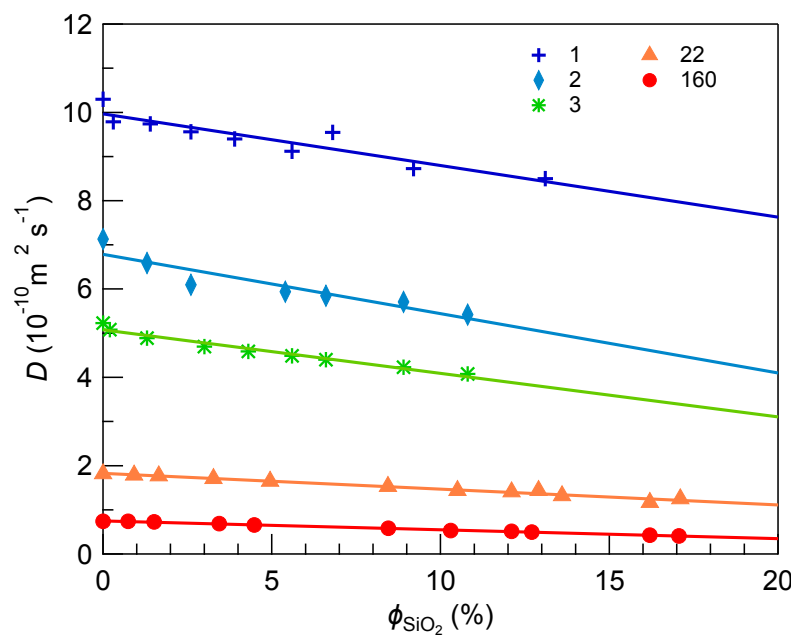

Figure 1: Diffusion coefficient of the propionate $(n=1,+)$, glutarate $(n=2$, $\checkmark), 1,3,5$-pentanetricarboxylate $(n=3, *)$ and polyacrylate $(n=22, \boldsymbol{\Delta}, n=$ $160, \bullet)$ versus the volume fraction of silica $\mathrm{LS}$ at $\mathrm{pH}=8$.

As shown in Fig. 11. the increase of the volume fraction of obstacles causes a linear decrease of the diffusion coefficient of all studied diffusers for the range of volume fraction studied. The data in Fig. 1 can then been fitted with a linear dependence of the diffusion coefficient (equation 1 ). 
The parameter $\alpha$ equals 0.5 in the case of the linear approximation of the pure obstruction model in the limit $R_{\mathrm{p}} \ll R_{\mathrm{o}}$, but it is close to 2 for colloidal particles of the same size. In the first row of Table 1 , the slope $\alpha$ of the linear fit are reported (the errors are the standard deviation values issued from the fitting procedure).

The deviation of the data from the pure obstruction model $(\alpha=0.5)$ increases with the molecular weight of the diffusers, as the increase of the parameter $\alpha$ reported in Table 1]points out. Jönsson et al. have suggested [27] for an analogous system, that the difference might be due to properties such as the size of the diffuser and the interaction between diffusers and obstacles, that are not considered in their model. Both effects become more significant as the volume fraction of obstacles increases and the size of diffuser increases. For the oligomers however, the minimum size ratio is of the order of $R_{\mathrm{p}} / R_{\mathrm{o}} \sim 1 / 16$ which means that the interaction effect is the most important.

No apparent difference in the relative slope $\alpha$ is observed for $n=2$ and $n=3$. The interaction between the diffuser and the obstacles seems unchanged despite the increase of the charge of the diffuser. This observation may be ascribed to the effect of the electrostatic condensation for the largest of the three oligomers. The maximum effective charge for the three oligomers can been estimated using the formula proposed by Ramanathan, who used Poisson-Boltzmann equation in the limit of small radius compared with the Debye length $\kappa R_{\mathrm{p}} \ll$ 1 [65, 66].

$$
Z_{\text {max }}=\frac{2 R_{\mathrm{p}}}{\ell_{B}} \ln \frac{1}{\kappa R_{\mathrm{p}}}
$$

The values found are respectively $Z_{\max }=1.4,1.6$, and 1.7, which can be compared with the structural charges (1,2 and 3 respectively). For the two smallest molecules, the structural charges are close to the calculated maximum charge but for the latter a significant decrease is observed leading to a $Z_{\max }$ for $n=3$ very close to the $n=2$ case due to a significant counterion condensation that can be expected due to its high charge density. The effective interaction for $n=3$ and $n=2$ diffusers and the obstacles are therefore similar which is the reason why their diffusion decrease does not noticeably differ.

\subsection{Comparison with the cell-model}

In this paragraph, we apply the cell model with the screened Coulomb potential (SCP) to estimate the diffusion of the various carboxylate molecules with the charge of silica particles at $\mathrm{pH}=8$. The volume fraction of obstacles varied between 0 and 0.2 and the slope of the diffusion coefficient was determined and compared with the experimental results of Table 1.

The Debye length used for the calculation was $\kappa^{-1}=2 \mathrm{~nm}$. The simulated system was at $\mathrm{pH}=8$, where the charge of the silica nanoparticles of radius $R_{\mathrm{o}}=8 \mathrm{~nm}$ is $Z_{\mathrm{eff}}=-119$ (see supplementary material for the determination). The charge of the diffusers are respectively $z=-1$, for the propanoate ion, $z=-2$ for the glutarate ion and $z=-3$ for the $1,3,5-$ pentanetricarboxylate ion. As mentioned before, these ions are considered to be point-like $\left(R_{\mathrm{p}} \simeq 0\right)$. For the polyelectrolytes, the effective charge was calculated taking into account the effect of the condensation of counterion to yield the following

\begin{tabular}{cccccc}
\hline$n$ & 1 & 2 & 3 & 22 & 160 \\
\hline$\alpha_{\exp }$ & $1.2 \pm 0.2$ & $1.9 \pm 0.2$ & $1.9 \pm 0.2$ & $2.0 \pm 0.1$ & $2.7 \pm 0.1$ \\
$\alpha_{\text {SCP }}$ & $0.99 \pm 0.02$ & $1.37 \pm 0.02$ & $1.57 \pm 0.02$ & $2.43 \pm 0.04$ & $5.51 \pm 0.07$ \\
\hline
\end{tabular}

Table 1: Slope $\alpha$ of the linear fit for experimental data and the calculated data with the cell model with screened Coulomb potential (SCP) for various polyacrylate lengths ( $n$ is the degree of polymerization).

parameters PAA $n=22: z=-8$ and $R_{\mathrm{p}}=1.2 \mathrm{~nm}$; PAA $n=160: z=-53$ and $R_{\mathrm{p}}=2.6 \mathrm{~nm}$ [56].

The values of the initial slopes calculated by this method $\alpha_{\mathrm{SCP}}$ are reported in Table 1, together with the slopes extracted by the linear fit of the experimental data $\alpha_{\text {exp }}$.

The cell model with the screened Coulomb potential gives the correct trend and order of magnitude of the deviation of the diffusion coefficient. However, the agreement is only semiquantitative since the model fails to satisfactorily reproduce the values of slope observed. The computed correction is too weak for the small molecules but too large for the larger polyelectrolyte. The smaller diffusers are able to more easily explore the region where the screened Coulomb potential approximation fails, that is to say where the real electrostatic potential is higher than the linearized Poisson-Boltzmann version. For these ions, the use of the MPB potential as suggested by Bratko and Lindman [55] seems more suitable. For the larger diffusers, additional effects can be involved, since these particles are not only larger but, also flexible which might enhance their ability to diffuse. Finally, their diffusion coefficient is not so small compared to the one of the obstacles.

Even if the cell-model with the screened Coulomb potential does not achieve an entirely satisfying description of the observed results, it is still a valuable benchmark. In what follows, we will vary the other parameters of the interaction potential to assess their respective influence.

\subsection{Changing the interaction}

To further explore the role of the interactions, we will now modulate the electrostatic repulsion. The addition of salt acts on the range of the repulsion and the change of $\mathrm{pH}$ affects the amplitude.

\subsubsection{Screening by salt addition}

The electrostatic interaction can be screened with salt addition, hence reducing the range of the electrostatic repulsion by decreasing $\kappa^{-1}$. The diffusion coefficients of the PAANa ( $n=22$ and $n=160$ ) are studied as a function of the volume fraction of obstacles, in the presence of an added monovalent salt, $\mathrm{NaCl}$. In Fig. 2, the diffusion coefficients of the PAA $(n=160)$ with $0.1 \mathrm{~mol} \mathrm{~L}^{-1}$ of added $\mathrm{NaCl}$ are reported as a function of the volume fraction of silica LS together with the diffusion coefficients without added salt. The diffusion coefficient exhibit higher values at $0.1 \mathrm{~mol} \mathrm{~L}^{-1}$, than the system without added salt. For all volume fraction of obstacles, the self-diffusion of the PAANa is higher in the presence of salt. Because the salt is screening the electrostatic interactions in the system, the effect of the salt on the PAANa intra-chain interactions, have to be separated from the effects between PAANa and silica nanoparticles.

Without obstacles, the increase of the diffusion coefficient is caused by the screening of the intra-chain interactions of the 


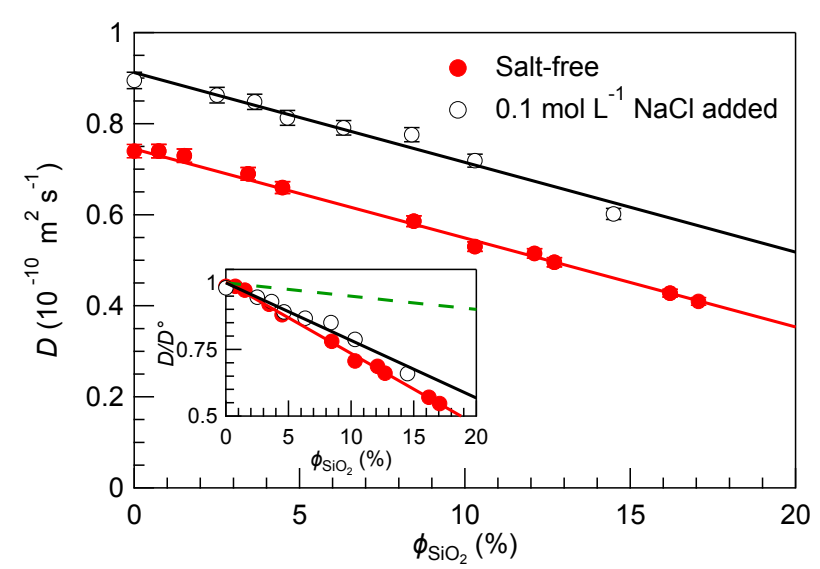

Figure 2: Diffusion coefficients of the PAANa $(n=160$, concentration $0.7 \mathrm{wt} \%$ ) versus the volume fraction of silica in a salt-free solution $(\bullet)$, and $0.1 \mathrm{~mol} \mathrm{~L}^{-1} \mathrm{NaCl}(\mathrm{\circ})$. Inset: relative diffusion coefficient. The dashed line is the pure obstruction model (equation 1). $T=298 \mathrm{~K}, \mathrm{pH} \approx 8$.

PAANa, which shrinks the chain otherwise stretched by the electrostatic repulsion. However, in the presence of obstacles, we have to consider the effect of the salt on the diffuser-obstacle interaction.

To isolate the effect on the dynamics caused by the screening of the polyelectrolyte-silica interaction, the data have been normalized by the diffusion coefficient without obstacles (see Fig. 2 inset) and fitted as before to extract the slope. In the inset of Fig. 2. an increase of the diffusion coefficient of the polyelectrolyte in the presence of added salt is observed. The relative diffusion coefficient with $0.1 \mathrm{~mol} \mathrm{~L}^{-1}$ of added salt get closer but is still far from the pure obstruction behaviour (dashed line).

The values of the slopes $\alpha$, extracted from the fit, are reported in Table 2 for the longer chains. For both polyelectrolyte sizes, the slope $\alpha$ decreases with the addition of salt, i.e. the diffusion coefficient of the PAANa increases. When salt is added, the effective range of the electrostatic repulsion, the Debye length $\kappa^{-1}$, is reduced from $1.7 \mathrm{~nm}$ without added salt down to $0.95 \mathrm{~nm}$ for $0.1 \mathrm{~mol} \mathrm{~L}^{-1} \mathrm{NaCl}$.

\begin{tabular}{ccccc}
\hline$n$ & \multicolumn{2}{c}{22} & \multicolumn{2}{c}{160} \\
\hline added salt $\left(\mathrm{mol} \mathrm{L}^{-1}\right)$ & $\alpha_{\exp }$ & $\alpha_{\mathrm{SCP}}$ & $\alpha_{\exp }$ & $\alpha_{\mathrm{SCP}}$ \\
\hline 0 & $1.9 \pm 0.1$ & $2.43 \pm 0.04$ & $2.7 \pm 0.1$ & $5.51 \pm 0.07$ \\
0.1 & $1.3 \pm 0.1$ & $1.22 \pm 0.01$ & $2.2 \pm 0.2$ & $2.43 \pm 0.03$ \\
\hline
\end{tabular}

Table 2: Slopes, $\alpha$ of the linear fit (equation. 1 of the experimental diffusion coefficient with or without added salt and the calculated diffusion with the cell model with screened Coulomb potential (SCP).

The slopes were also calculated with the SCP cell-model where the screening length was changed to $\kappa^{-1}=0.95 \mathrm{~nm}$, while keeping the other parameters constants. The corresponding slopes are reported in Table 2. The decrease of the Debye length indeed makes the diffusion of polyelectrolyte faster thanks to the diminished range of repulsion. In addition, for this highly screened regime the agreement of the slopes calculated with the SCP cell-model and the experimentally measured one improves. Finally, even with this notable concentration of salt added and the ensuing screening of the interaction, its consequence on the diffusion still differs significantly from the pure obstruction.

To investigate further the effect of the salt addition, the diffusion coefficient of the PAANa $(n=160)$, at a fixed obstacle concentration $(\phi=9 \%)$, has been studied as a function of the added salt concentration (Fig. 3). As in the polyelectrolyte suspension without obstacles (data also reported in Fig. 3), the addition of salt enhances the diffusion coefficient of the polyelectrolyte. However, in the presence of silica particles, the increase of the diffusion coefficient is slightly higher than in the absence of silica obstacles, since the silica-PAANa repulsions are also screened.

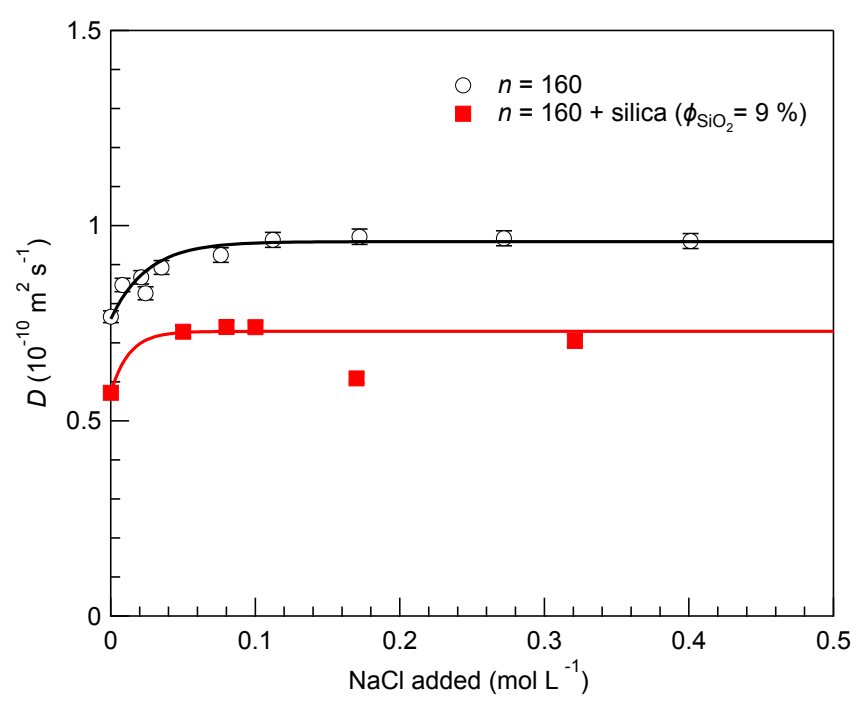

Figure 3: Diffusion coefficients of the PAANa $(n=160$, concentration $1.5 \mathrm{wt} \%)$ in a silica LS suspension $(\phi=9 \%)$, as a function of the molarity of $\mathrm{NaCl}$ of the solution. The lines are guides to the eye. $T=298 \mathrm{~K}, \mathrm{pH} \approx 8$.

The main change in the diffusion coefficient occurs below $0.1 \mathrm{~mol} \mathrm{~L}^{-1}$ of added salt where the ratio between the screening length $\kappa^{-1}$ and the radius of the obstacle $R_{\mathrm{o}}$ is higher. For larger concentrations of added salt, a plateau behavior is observed since the diffuse layer of ions around the particle tends to be thin compared to the diameter of the particle $\left(\kappa R_{\mathrm{o}} \gg 1\right)$.

The impact of the addition of salt on the diffusion of the propanoate in the presence of silica nanoparticles was studied with a similar procedure (data not shown). For this diffuser, equivalent to one monomer of the polyacrylate, no intramolecular interactions are present and only the effect of salt addition on diffuser-obstacle interaction can be investigated. It was observed that, contrary to the larger PAANa chains, the diffusion coefficient decreases with the addition of salt. This effect on the diffusion of the propanoate can be ascribed to the interaction with the added electrolyte [67] which seems to be larger than the effect of the change of screening length around the obstacles.

\subsubsection{Changing the charges with $p H$}

In this section, the charge of the particles and diffusers are modified via the change of the $\mathrm{pH}$ value of the suspension. As a result, the predominant electrostatic repulsion at high $\mathrm{pH}$ values can be progressively weakened by decreasing the $\mathrm{pH}$ to reach a state of uncharged obstacles and diffusers for low $\mathrm{pH}$ 
values. For these low $\mathrm{pH}$ values, the regime of pure obstruction is expected if no specific adsorption occurs.

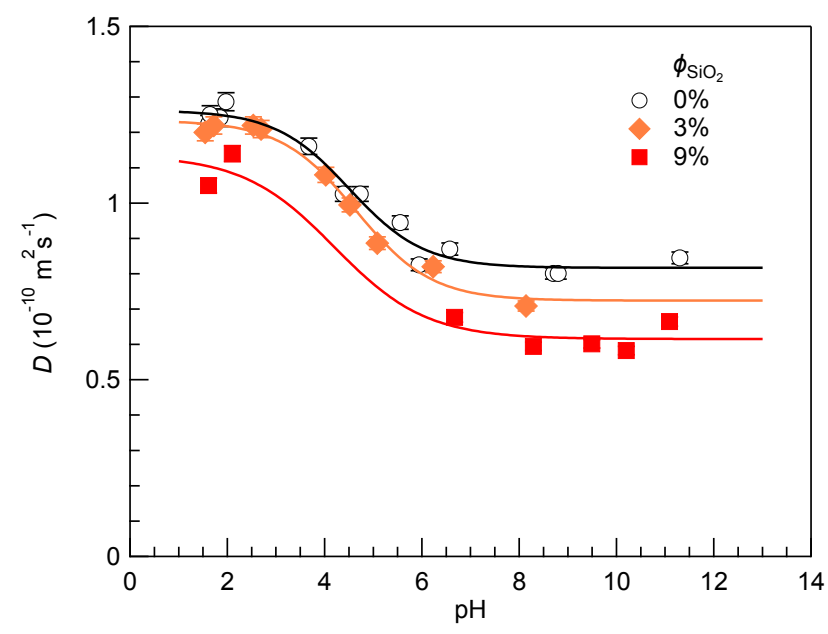

Figure 4: Diffusion coefficient of PAANa $(n=160, w \sim 0.7 \%)$ vs $\mathrm{pH}$, without obstacles (०), with silica LS $(\phi=3 \% \bullet)$, and silica LS $(\phi=9 \% \mathbf{\square}) . T=$ $298 \mathrm{~K}$.

In Fig. 4, the diffusion coefficient of the PAANa $(n=160)$ as a function of the $\mathrm{pH}$ is reported for three systems: without obstacles, and with obstacles at two volume fractions $\phi=3 \%$ and $\phi=9 \%$. All the diffusion coefficients exhibit a sigmoidal behaviour with the $\mathrm{pH}$. As observed previously, the diffusion coefficient decreases with the concentration of obstacles whatever the value of $\mathrm{pH}$. This sigmoidal shape is present even in the absence of obstacles due to the change of conformation of the polyelectrolyte with its protonation state depending on the $\mathrm{pH}$ value. This change was extensively studied in a previous study [56]. For a fixed volume fraction of obstacles, the decreases of the diffusion coefficient depends on the $\mathrm{pH}$ value: it is stronger at high $\mathrm{pH}$ (close to $25 \%$ for $\phi=9 \%$ ), and achieves the lowest value at low $\mathrm{pH}$ (around $10 \%$ for $\phi=9 \%$ ).

At high $\mathrm{pH}$, the charged diffuser is repulsed by a charged obstacle with like charge, whereas at low $\mathrm{pH}$ values, the diffuser is mainly affected by the physical volume of the obstacle, since both the diffuser and the obstacle are uncharged.

In the low $\mathrm{pH}$ limit, the diffusion coefficient in the presence of the silica nanoparticles decreases by $2 \%$ for $\phi=3 \%$ and by $11 \%$ for $\phi=9 \%$, compared to the value of the diffusion coefficient without nanoparticles. In this $\mathrm{pH}$ range, the electrostatic repulsion vanishes but due to its own size the polyelectrolyte center of mass cannot explore all the volume. A simple way to take into account this effect is to rescale the volume fraction into an effective volume fraction corresponding to the volume accessible to the center of mass of the diffuser as $\phi^{\prime}=\phi\left(1+R_{\mathrm{p}} / R_{\mathrm{o}}\right)^{3}$ where $R_{\mathrm{p}}$ is the radius of the diffuser and $R_{\mathrm{o}}$ the radius of the nanoparticle. We can use a rescaled version of equation 1, $D=D^{\circ}\left[1-\frac{1}{2} \phi^{\prime}\right]$, to compute the decrease of diffusion in the hypothesis of a pure obstruction. At $\mathrm{pH}=2$, the radius of the PAA with $n=160$ is $1.8 \mathrm{~nm}$ [56] and the computed decrease are respectively $2.7 \%$ for $\phi=3 \%$ and $8.2 \%$ for $\phi=9 \%$. The diffusion coefficient is then in fair agreement to the pure obstruction behavior. Besides, a weak adsorption at low $\mathrm{pH}$ of the neutral diffuser on the neutral surface of the particles cannot be completely ruled out. This adsorption is pre- vented at high $\mathrm{pH}$ by the strong electrostatic interaction [68].

\subsection{Going further with the SCP cell-model}

So far we have tried to apply the cell-model with the screened Coulomb potential to our experimental findings. This model can however be used in a wider range of systems. For example, in Fig. 5, the relative diffusion coefficient of a pointlike ion of charge $z=-1$ was computed as a function of the effective charge of the obstacle $Z_{\text {eff }}$ and a volume fraction of $10 \%$. For an uncharged particle, the pure obstruction behavior is observed, but according to the sign of the charge the change of the diffusion coefficient differs strongly.

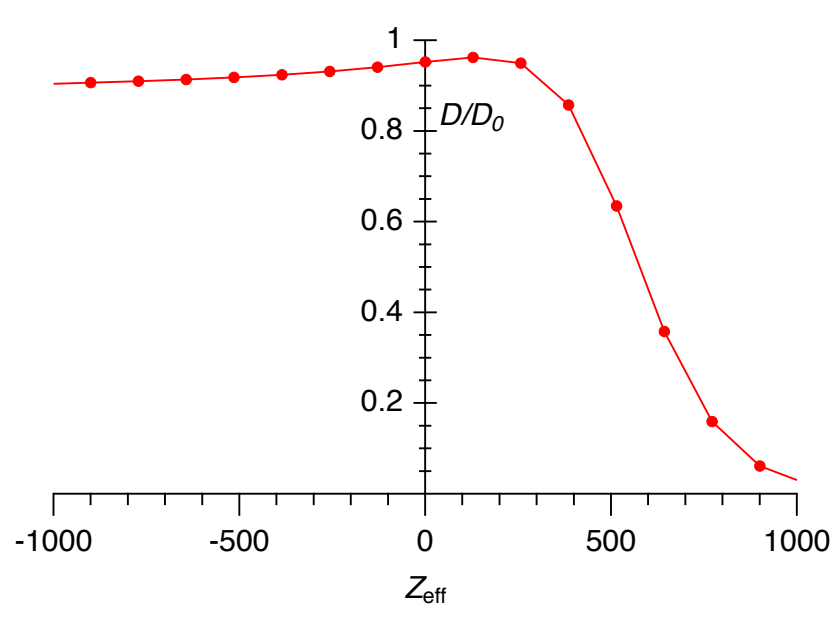

Figure 5: Relative diffusion coefficient of a point-like anion $(z=-1), \kappa=$ $1 \mathrm{~nm}^{-1}, R_{\mathrm{O}}=8 \mathrm{~nm}$ and $\phi=10 \%$.

For co-ion $\left(Z_{\mathrm{eff}}<0\right)$, a monotonous and rather moderate decrease is observed. This was the domain studied in our samples where the diffuser and the obstacles bear charges of the same sign. The decrease can then be explained by the increase of the repulsion with the increase of the absolute value of $Z_{\text {eff }}$. For counter-ions $\left(Z_{\text {eff }}>0\right)$, the evolution of the relative diffusion coefficient with the charge of the obstacle appears to be completely different: for low $Z_{\text {eff }}$ there is small increase of the diffusion coefficient, followed by a steep decrease. This nonmonotonous dependence can be explained as follows. When a small attraction is present (small $Z_{\text {eff }}$ ) the probability of having a diffuser close to the surface of the obstacles increases and the diffusion paths are then shorter, i.e. the diffusion is faster. If the attraction is higher, the diffuser is trapped in the vicinity of the obstacle and the diffusion is strongly hindered. Such nonmonotonous behavior has already been observed for the diffusion of ion in nanoporous media [21] or for the diffusion of particle among larger attractive spheres [20]. Even if the investigation of this behavior is appealing, the experimental preparation of systems where significant but small attraction exists is difficult since the colloidal stability is no longer ensured. In addition, for diffusers close to solid surfaces, the faster spin relaxation makes the determination of small diffusion coefficients by NMR difficult. 


\section{Conclusion}

The addition of charged obstacles in a polyelectrolyte suspension causes a linear decrease of the diffusion coefficient of the polyelectrolyte for volume fractions of obstacles below $20 \%$. The observed decrease is higher for larger and more charged particles. Of the two effects responsible for this decrease, obstruction and interaction, the major one is the repulsive electrostatic interaction. It is clearly evidenced by the effect of a change of salt concentration or a change of $\mathrm{pH}$ value of the suspension. The resolution of a Smoluchowski equation for the diffusion in a screened Coulomb potential with the use of a cell model derived from [48], yield only a semi-quantitative agreement with the experimental results. The model predicts the trend and the effect of salt addition. Therefore, a more elaborate description is required to fully reproduce the phenomenon which would include, for example, the dynamics of the obstacles and the resulting hydrodynamic effect or even the internal flexibility of the diffusers. Such descriptions are unfortunately very demanding due to their complexity. However, the use of a simpler cell model description is still a promising tool and benchmark to investigate the effect of interaction on the diffusion in crowded media. It gives some hints to design experimental systems even if they are challenging to prepare.

\section{Acknowledgements}

This study was funded by Sorbonne University. The authors thank Dr. Isabelle Correia (Sorbonne University) for the NMR facilities and technical assistance.

\section{References}

[1] P. Bernadó, J. G. de la Torre, M. Pons, Macromolecular crowding in biological systems: hydrodynamics and NMR methods, J. Mol. Recognit. 17 (2004) 397-407. doi 10.1002/jmr.694

[2] R. Pecora, Dynamics of Biological Polyelectrolytes, Macromol. Symp. 229 (2005) 18-23. doi 10.1002/masy . 200551103

[3] G.-W. Li, O. G. Berg, J. Elf, Effects of macromolecular crowding and DNA looping on gene regulation kinetics, Nat. Phys. 5 (2009) 294-297. doi $10.1038 /$ nphys 1222

[4] S. R. McGuffee, A. H. Elcock, Diffusion, Crowding \& Protein Stability in a Dynamic Molecular Model of the Bacterial Cytoplasm, PLoS Comput. Biol. 6 (2010) e1000694. doi 10.1371/journal.pcbi.1000694

[5] C. E. J. Dieteren, S. C. A. M. Gielen, L. G. J. Nijtmans, J. A. M. Smeitink, H. G. Swarts, R. Brock, P. H. G. M. Willems, W. J. H. Koopman, Solute diffusion is hindered in the mitochondrial matrix, Proc. Natl. Acad. Sci. 108 (2011) 8657-8662. doi 10.1073/pnas.1017581108

[6] I. Pastor, L. Pitulice, C. Balcells, E. Vilaseca, S. Madurga, A. Isvoran, M. Cascante, F. Mas, Effect of crowding by Dextrans in enzymatic reactions, Biophys. Chem. 185 (2014) 8-13. doi 10.1016/j .bpc. 2013. 10.006

[7] C. Di Rienzo, V. Piazza, E. Gratton, F. Beltram, F. Cardarelli, Probing short-range protein Brownian motion in the cytoplasm of living cells, Nat. Commun. 5 (2014) 5891. doi 10.1038/ncomms6891

[8] M. Feig, I. Yu, P.-h. Wang, G. Nawrocki, Y. Sugita, Crowding in Cellular Environments at an Atomistic Level from Computer Simulations, J. Phys. Chem. B 121 (2017) 8009-8025. doi 10.1021/acs.jpcb.7b03570

[9] P. A. A. Ignacio-de Leon, Y. Eygeris, R. Haynes, I. Zharov, Diffusion of Proteins across Silica Colloidal Crystals, Langmuir (2018) acs.langmuir.8b01261. doi 10.1021/acs.langmuir.8b01261

[10] C.-C. Lin, E. Parrish, R. J. Composto, Macromolecule and Particle Dynamics in Confined Media, Macromolecules 49 (2016) 5755-5772. doi 10.1021/acs.macromol.6b00471
[11] X.-W. Huang, Y. Peng, J.-h. Huang, M.-b. Luo, A study on the diffusivity of polymers in crowded environments with periodically distributed nanoparticles, Phys. Chem. Chem. Phys. 19 (2017) 2997529983. doi 10.1039/C7CP05514E

[12] E. Bartsch, Diffusion in concentrated colloidal suspensions and glasses, Curr. Opin. Colloid Interface Sci. 3 (1998) 577-585. doi 10.1016/ S1359-0294(98) 80083-6

[13] Q. Berrod, S. Hanot, A. Guillermo, S. Mossa, S. Lyonnard, Water subdiffusion in membranes for fuel cells, Sci. Rep. 7 (2017) 8326. doi 10 . 1038/s41598-017-08746-9

[14] T. Brenner, S. Matsukawa, Anomalous diffusion of poly(ethylene oxide) in agarose gels, Int. J. Biol. Macromol. 92 (2016) 1151-1154. doi 10. 1016/j.ijbiomac.2016.07.054

[15] F. Babayekhorasani, D. E. Dunstan, R. Krishnamoorti, J. C. Conrad, Nanoparticle diffusion in crowded and confined media, Soft Matter 12 (2016) 8407-8416. doi 10.1039/C6SM01543C

[16] P. Nath, R. Mangal, F. Kohle, S. Choudhury, S. Narayanan, U. Wiesner, L. A. Archer, Dynamics of Nanoparticles in Entangled Polymer Solutions, Langmuir 34 (2018) 241-249. doi 10.1021/acs.langmuir. $7 \mathrm{~b} 03418$

[17] C. Roberts, T. Cosgrove, R. G. Schmidt, G. V. Gordon, Diffusion of Poly(dimethylsiloxane) Mixtures with Silicate Nanoparticles, Macromolecules 34 (2001) 538-543. doi 10.1021/ma001245z

[18] D. A. Tsehay, M. Luo, Static and dynamic properties of a semiflexible polymer in a crowded environment with randomly distributed immobile nanoparticles, Phys. Chem. Chem. Phys. 20 (2018) 9582-9590. doi 10. 1039/C7CP08341F

[19] S.-J. E. Lee, A. K. Chakraborty, Sequence dependence of polymer dynamics in quenched disordered media: Weak attraction facilitates transport, J. Chem. Phys. 117 (2002) 10869-10876. doi 10.1063/1. 1519838

[20] G. G. Putzel, M. Tagliazucchi, I. Szleifer, Nonmonotonic Diffusion of Particles Among Larger Attractive Crowding Spheres, Phys. Rev. Lett. 113 (2014) 138302. doi 10.1103/PhysRevLett.113.138302

[21] M. Jardat, B. Hribar-Lee, V. Vlachy, Self-diffusion of ions in charged nanoporous media, Soft Matter 8 (2012) 954. doi 10.1039/ c1sm05985h

[22] P. M. Kekenes-Huskey, C. E. Scott, S. Atalay, Quantifying the Influence of the Crowded Cytoplasm on Small Molecule Diffusion, J. Phys. Chem. B 120 (2016) 8696-8706. doi 10.1021/acs .jpcb.6b03887

[23] W. S. Price, NMR Studies of Translational Motion, 1 ed., Cambridge University Press, 2009.

[24] P. T. Callaghan, Translational Dynamics and Magnetic Resonance: Principles of Pulsed Gradient Spin Echo NMR, Oxford University Press, Oxford, 2011.

[25] P. G. de Gennes, Reptation of a Polymer Chain in the Presence of Fixed Obstacles, J. Chem. Phys. 55 (1971) 572. doi 10.1063/1.1675789

[26] H. Lekkerkerker, J. Dhont, On the calculation of the self-diffusion coefficient of interacting Brownian particles, J. Chem. Phys. 80 (1984) 57905792. doi $10.1063 / 1.446602$

[27] B. Jönsson, H. Wennerström, P. G. Nilsson, P. Linse, Self-diffusion of small molecules in colloidal systems, Colloid Polym. Sci. 264 (1986) 77-88. doi 10.1007/BF01410310

[28] A. Baumgärtner, M. Muthukumar, A trapped polymer chain in random porous media, J. Chem. Phys. 87 (1987) 3082-3088. doi 10.1063/1. 453045

[29] M. Muthukumar, A. Baumgärtner, Diffusion of a polymer chain in random media, Macromolecules 22 (1989) 1941-1946. doi 10.1021/ ma00194a071

[30] E. Vilaseca, A. Isvoran, S. Madurga, I. Pastor, J. L. Garcés, F. Mas, New insights into diffusion in 3D crowded media by Monte Carlo simulations: effect of size, mobility and spatial distribution of obstacles, Phys. Chem. Chem. Phys. 13 (2011) 7396. doi 10.1039/c0cp01218a

[31] A. H. Muhr, J. M. Blanshard, Diffusion in gels, Polymer (Guildf). 23 (1982) 1012-1026. doi 10.1016/0032-3861(82)90402-5

[32] J. K. G. Dhont, An introduction to the dynamics of colloids, Elsevier Science, 1996.

[33] G. Nägele, J. K. G. Dhont, G. Meier, in: P. Heitjans, J. Kärger (Eds.), Diffus. Condens. Matter, Springer-Verlag, Berlin/Heidelberg, 2005, pp. 620-715.

[34] M. Jardat, B. Hribar-Lee, V. Vlachy, Self-diffusion coefficients of ions in the presence of charged obstacles, Phys. Chem. Chem. Phys. 10 (2008) 449. doi $10.1039 / \mathrm{b} 711814 \mathrm{~g}$

[35] T. Ando, J. Skolnick, Crowding and hydrodynamic interactions likely 
dominate in vivo macromolecular motion., Proc. Natl. Acad. Sci. U.S.A. 107 (2010) 18457-18462. doi 10.1073/pnas.1011354107

[36] M. Długosz, J. Trylska, Diffusion in crowded biological environments: applications of Brownian dynamics., BMC Biophys. 4 (2011) 3. doi 10 . 1186/2046-1682-4-3

[37] T. Stylianopoulos, M.-Z. Poh, N. Insin, M. G. Bawendi, D. Fukumura, L. L. Munn, R. K. Jain, Diffusion of particles in the extracellular matrix the effect of repulsive electrostatic interactions., Biophys. J. 99 (2010) 1342-9. doi $10.1016 /$ j.bpj.2010.06.016

[38] C. Echeverria, R. Kapral, Macromolecular dynamics in crowded environments, J. Chem. Phys. 132 (2010) 104902. doi 10.1063/1.3319672

[39] A. Tomilov, A. Videcoq, T. Chartier, T. Ala-Nissilä, I. Vattulainen, Tracer diffusion in colloidal suspensions under dilute and crowded conditions with hydrodynamic interactions., J. Chem. Phys. 137 (2012) 014503. doi $10.1063 / 1.4731661$

[40] G. Nägele, P. Baur, Long-time dynamics of charged colloidal suspensions: hydrodynamic interaction effects, Physica A 245 (1997) 297-336. doi 10.1016/S0378-4371(97)00307-5

[41] M. Jardat, O. Bernard, C. Treiner, P. Turq, Electrical Conductance of Cryptate Solutions: Comparison between Experiments and Simulation Results, J. Phys. Chem. B 103 (1999) 8462-8466. doi 10.1021/ jp991350e

[42] G. Batôt, V. Dahirel, G. Mériguet, a. a. Louis, M. Jardat, Dynamics of solutes with hydrodynamic interactions: Comparison between Brownian dynamics and stochastic rotation dynamics simulations, Phys. Rev. E 88 (2013) 043304. doi 10.1103/PhysRevE. 88.043304

[43] G. Nägele, M. Medina-Noyola, J. L. Arauz-Lara, R. Klein, in: New Trends Colloid Sci., volume 73 of Progress in Colloid E Polymer Science, Steinkopff, Darmstadt, 1987, pp. 5-9.

[44] J. L. Arauz-Lara, H. Ruiz-Estrada, M. Medina-Noyola, G. Nägele, R. Klein, in: Trends Colloid Interface Sci. V, volume 84 of Progress in Colloid E Polymer Science, Steinkopff, Darmstadt, 1991, pp. 377-380.

[45] J. L. Arauz-Lara, H. Ruiz-Estrada, M. Medina-Noyola, Tracer Diffusion in Colloidal Mixtures, J. Colloid Interface Sci. 171 (1995) 127-133. doi $10.1006 /$ jcis.1995.1158

[46] G. Nägele, J. K. G. Dhont, Tracer-diffusion in colloidal mixtures: A mode-coupling scheme with hydrodynamic interactions, J. Chem. Phys. 108 (1998) 9566. doi $10.1063 / 1.476405$

[47] J. H. Wang, Theory of the Self-diffusion of Water in Protein Solutions. A New Method for Studying the Hydration and Shape of Protein Molecules, J. Am. Chem. Soc. 76 (1954) 4755-4763. doi 10.1021/ja01648a001

[48] G. M. Bell, Self-diffusion of ions in the electric fields of spherical particles, Trans. Faraday Soc. 60 (1964) 1752. doi 10.1039/tf9646001752

[49] P. Venema, R. Struis, J. Leyte, D. Bedeaux, The effective self-diffusion coefficient of solvent molecules in colloidal crystals, J. Colloid Interface Sci. 141 (1991) 360-373. doi 10.1016/0021-9797(91) 90332-3

[50] G. M. Bell, A. J. Dunning, Counter-ion self-diffusion in a concentrated micellar system, Trans. Faraday Soc. 66 (1970) 500. doi 10.1039/ tf9706600500

[51] D. Chan, B. Halle, The Smoluchowski-Poisson-Boltzmann description of ion diffusion at charged interfaces, Biophys. J. 46 (1984) 387-407. doi 10.1016/S0006-3495(84)84035-7

[52] L. Belloni, M. Drifford, P. Turq, Counterion diffusion in polyelectrolyte solutions, Chem. Phys. 83 (1984) 147-154. doi 10.1016/ 0301-0104(84) 85229-5

[53] L. G. Nilsson, L. Nordenskiöld, P. Stilbs, W. H. Braunlin, Macroscopic counterion diffusion in solutions of cylindrical polyelectrolytes, J. Phys. Chem. 89 (1985) 3385-3391. doi 10.1021/j100261a045

[54] N. Fatin-Rouge, A. Milon, J. Buffle, R. R. Goulet, A. Tessier, Diffusion and Partitioning of Solutes in Agarose Hydrogels: The Relative Influence of Electrostatic and Specific Interactions, J. Phys. Chem. B 107 (2003) 12126-12137. doi 10.1021/jp0303164

[55] D. Bratko, B. Lindman, On counterion self-diffusion in micellar solutions, J. Phys. Chem. 89 (1985) 1437-1440. doi 10.1021/ j100254a027

[56] C. Dolce, G. Mériguet, Ionization of short weak polyelectrolytes: when size matters, Colloid Polym. Sci. 295 (2017) 279-287. doi 10.1007/ s00396-016-4000-x

[57] D. Wu, A. Chen, C. Johnson, An Improved Diffusion-Ordered Spectroscopy Experiment Incorporating Bipolar-Gradient Pulses, J. Magn. Reson. A 115 (1995) 260-264. doi 10.1006/jmra.1995.1176

[58] L. G. Longsworth, THE MUTUAL DIFFUSION OF LIGHT AND HEAVY WATER, J. Phys. Chem. 64 (1960) 1914-1917. doi 10.1021/ j100841a027
[59] M. Holz, H. Weingärtner, Calibration in accurate spin-echo self-diffusion measurements using $1 \mathrm{H}$ and less-common nuclei, J. Magn. Reson. 92 (1991) 115-125. doi 10.1016/0022-2364(91) 90252-0

[60] V. Dahirel, M. Jardat, Effective interactions between charged nanoparticles in water: What is left from the DLVO theory?, Curr. Opin. Colloid Interface Sci. 15 (2010) 2-7. doi 10.1016/j . cocis .2009.05.006

[61] Maple 2015, Maplesoft, a division of Waterloo Maple Inc., Waterloo, Ontario, 2015.

[62] D. S. Banks, C. Fradin, Anomalous diffusion of proteins due to molecular crowding, Biophys. J. 89 (2005) 2960-2971. doi 10.1529/biophysj . 104.051078

[63] P. Polanowski, A. Sikorski, Simulation of diffusion in a crowded environment., Soft Matter 10 (2014) 3597-607. doi 10.1039/c3sm52861h

[64] S. K. Ghosh, A. G. Cherstvy, R. Metzler, Non-universal tracer diffusion in crowded media of non-inert obstacles, Phys. Chem. Chem. Phys. 17 (2015) 1847-1858. doi 10.1039/C4CP03599B

[65] G. V. Ramanathan, Counterion condensation in micellar and colloidal solutions, J. Chem. Phys. 88 (1988) 3887. doi 10.1063/1.453837

[66] L. Belloni, Ionic condensation and charge renormalization in colloidal suspensions, Colloid Surf. A Phys. Eng. Asp. 140 (1998) 227-243. doi 10.1016/S0927-7757(97)00281-1

[67] L. Onsager, R. M. Fuoss, Irreversible Processes in Electrolytes. Diffusion, Conductance and Viscous Flow in Arbitrary Mixtures of Strong Electrolytes, J. Phys. Chem. 36 (1931) 2689-2778. doi 10.1021/ j150341a001

[68] C. Flood, T. Cosgrove, Y. Espidel, I. Howell, P. Revell, Sodium polyacrylate adsorption onto anionic and cationic silica in the presence of salts, Langmuir 23 (2007) 6191-6197. doi 10.1021/la070047z 